\title{
Phlébotomes de Touraine
}

\author{
par R. HOUIN, M. DENIAU, F. PUEL, F. REYNOUARD, \\ D. BARBIER et M. BONNET \\ (Collaboration technique: A. Plouton et L. Pontegnie) \\ Laboratoires de Parasitologie des Facultés de Médecine \\ de Ciéteil ( $\mathrm{P}^{\mathrm{r}}$ agr. R. Hourv), 6, ine du Général-Sartail, F 94000 Créteil \\ de Tours ( $\mathrm{P}^{\mathrm{r}}$ СоMBESCOT), 2 bis, boulevard Tonnellé, $F 37000$ Tours
}

\section{Résumé.}

Dans la région de Tours, l'existence d'un foyer de leishmaniose canine a conduit à étudier les Phlébotomes. 378 exemplaires ont été capturés appartenant à deux espèces : $P$. perniciosus (325 exemplaires) et $P$. mascittii (53 exemplaires). La première espèce semble ne pas dépasser, au Nord et à l'Est, la limite de l'aire de répartition du chêne pubescent en peuplements denses, qui constitue ainsi un précieux indicateur écologique. A l'intérieur de cette zone, la vallée du Loir est plus infestée que les régions voisines. P. mascittii, par contre, est trouvé partout, mais toujours en très faible abondance.

Le foyer leishmanien étudié se situe par conséquent au voisinage de la limite septentrionale au-delà de laquelle seuls des cas sporadiques peuvent apparaître, par suite de la faible densité des vecteurs.

\section{Summary.}

\section{Phlebotomes of Touraine (France).}

Phlebotomes from the neighbourhoods of Tours (Touraine, France) are studied here in a focus of canine leishmaniosis; 378 specimens were captured belonging to two species : $P$. perniciosus (325 specimens) and $P$. mascittii (53 specimens). In the North and East, the first species does not seem to exceed the geographical limits of dense populations of the pubescent oak, and this constitutes a useful ecological indication. Inside this area, the Loir Valley is more infested than the neighbouring countries. In return, $P$. mascittii is found everywhere, but in feeble density.

This leishmanian focus is then situated near the septentrional limit beyond which only sporadic cases may be detected because of the low density of the vectors. 
La découverte d'un foyer de leishmaniose canine dans la région de Tours a été, pour beaucoup, une surprise : en France, cette parasitose est en effet considérée somme méditerranéenne. Seuls des cas sporadiques avaient jusqu'alors été observés en dehors des foyers méridionaux, et rien ne permettait de prévoir la stabilité de la transmission dans une région aussi septentrionale. Pourtant, plusieurs travaux antérieurs avaient déjà signalé dans cette région l'existence de Phlébotomes: dès 1932, dans ieur révision des Phlébotomes de France, Langeron et Nitzulescu indiquent la présence de P. perniciosus dans la Mayenne et dans le Maine-et-Loire. En 1934, c'est à Richelieu, dans l'Indre-et-Loire, que Nitzulescu et Dollfus le trouvent. En 1936, Parrot constate sa présence dans l'Indre, tandis qu'en 1935 et 1937, Lavier le reconnaît sur des exemplaires provenant du Loiret. Plus récemment, Rageau le signale dans la Vienne et l'un de nous, avec Beaucournu, le retrouve dans la Mayenne, le Maine-et-Loire et la Sarthe. Dans ces deux dernières publications sont également mentionnées deux autres espèces : $P$. mascittii, dans la Vienne ; $P$. ariasi, dans la Sarthe et la Vienne.

Depuis longtemps aussi, on connaissait la possibilité de la survenue de cas sporadiques de leishmaniose, en dehors des foyers méridionaux de l'affection. Nombre d'auteurs en avaient publié des observations, qu'il s'agisse de leishmaniose canine (Henry et Bory, 1935 ; Prat, 1944 ; Guilhon, 1950-1951 ; Guilhon et Loge, 1950) ou de leishmaniose humaine (Pehu et Bertoye, 1931-1937; Castillon, 1937 ; Domergue, 1940 ; Claisse et coll., 1950 ; Auct, 1962 ; Hany et coll., 1974). Le rapprochement de ces cas et des captures de Phlébotomes décrites conduisait à incriminer $P$. perniciosus, voire $P$. mascittii, dans la transmission accidentelle de la maladie, à partir de sujets infestés importés de l'un des foyers connus.

La constatation d'une enzootie pérenne dans la région de Tours a complètement changé les données du problème: il ne s'agissait plus de cas transmis accidentellement, mais bien du maintien d'un foyer, et il fallait penser que les Phlébotomes devaient exister en abondance suffisante, au moins quelque temps dans l'année, pour retransmettre, chaque été, la maladie à de nouveaux chiens. La possibilité d'une transmission directe, envisageable à la rigueur au début de l'étude, disparaissait en effet dès lors qu'étaient dépistés des malades en dehors des meutes, comme ce fut le cas en 1973 et 1974 . Une étude quantitative des vecteurs s'imposait donc. Ce sont les résultats de la prospection effectuée en juillet-août 1974 qui seront exposés ici.

\section{Objectifs et Méthodes}

Cette première prospection, limitée dans le temps, ne pouvait guère prétendre qu'à un inventaire des espèces présentes dans la zone et, si possible, à une estimation de la densité. Mais il aurait été imprudent de se limiter aux seuls villages où avaient été trouvés des chiens leishmaniens, puisque ce foyer n'est pas encore délimité. Aussi les piégeages ont-il très largement débordé l'aire étroite connue comme abritant l'enzootie et se sont-ils étendus dans un rayon d'environ 50 kilomètres autour. 
Bien que quelques essais de chasse nocturne au capturateur aient été effectués (sans succès, peut-être à cause des conditions météorologiques), la méthode générale de capture adoptée a été celle des pièges adhésifs, telle qu'elle est décrite par Rioux et coll. dans la monographie «Epidémiologie des leishmanioses dans le sud de la France ». Il était en effet important de disposer d'une technique qui permette la comparaison des résultats obtenus non seulement d'une station à l'autre, mais aussi avec ceux des piégeages effectués ailleurs. Les pièges (feuilles de papier de $20 \times 20 \mathrm{~cm}$, imprégnées d'huile de ricin) étaient disposés dans des anfractuosités et laissés en place cinq jours. Il n'a pas été fait usage de pièges éclairés. 3200 feuilles, soit $128 \mathrm{~m}^{2}$, ont été ainsi mises en place, en 177 stations. Dans la mesure du possible, les barbacanes ont été préférées pour disposer les pièges. Néanmoins, leur rareté, en particulier dans les zones dépourvues de relief, a souvent amené à piéger d'autres types de gîtes potentiels (trous de murs ou de rochers, bâtiments, caves troglodytes, assez fréquentes dans la région).

La région prospectée est un plateau de faible altitude $(100 \mathrm{~m})$, compris entre les vallées de la Loire au sud, et du Loir au nord et à l'ouest. Très boisé dans sa partie ouest, il s'ouvre largement à l'est sur la Beauce et change alors totalement de physionomie : zone de culture céréalière extensive. Outre ce plateau ont été échantillonnées les deux vallées, mais aussi une extension au sud de la Loire, entre celle-ci et la Vienne. Cette région est encore différente ; sans relief notable, elle est essentiellement vouée à l'élevage ou à des vergers.

L'étude des cartes de végétation de la région avait permis de constater le passage, dans le nord de la zone prospectée, à proximité de la vallée du Loir, de la limite septentrionale du chêne pubescent (Quercus lanuginosa, Lansk) en peuplements denses. L'intérêt de cette espèce comme indicateur écologique a été abondamment démontré au cours des travaux effectués dans le Midi par Rioux et coll., et il était intéressant de voir si, dans cette région aussi, existait une différence entre les stations situées en deçà et celles placées au-delà de cette limite. Pour cette raison, plusieurs stations ont été échantillonnées au nord de cette frontière, tout en sachant qu'une limite de ce type, dans une région de faible relief, ne pouvait qu'être approximative.

\section{Résultats}

378 Phlébotomes ont été capturés et déterminés, ce qui représente, compte tenu de la surface de piégeage $\left(128 \mathrm{~m}^{2}\right)$, une densité de $2,95 / \mathrm{m}^{2}$. Ce chiffre est à rapprocher de celui que donnent Rioux et coll. : $34 / \mathrm{m}^{2}$ pour le Midi. Globalement donc, la densité des captures est très faible.

Le détail des captures s'établit comme suit :

Phlebotomus perniciosus: 290 o 35 우

Phlebotomus mascittii : $\quad 18$ क 35 우 
On constate immédiatement l'absence de quatre des espèces françaises, et en particulier de Phlebotomus ariasi, pourtant antérieurement signalé dans une station située au nord de la zone étudiée (Juigné-sur-Sarthe, Houin et Beaucournu, 1966). Une explication vraisemblable est fournie par l'existence en ce point d'une situation climatique privilégiée, objectivée par la présence d'un peuplement de chêne vert, qui ne se retrouve nulle part ailleurs dans la région. Malgré l'abondance des Reptiles dans les biotopes étudiés, $S$. minuta n'a pas été trouvée, ce qui cadre avec l'aire de répartition purement méditerranéenne de cette espèce.

Deux espèces se partagent donc inégalement les captures, $P$. perniciosus représen$\operatorname{tant} 86 \%$ du total. Les densités s'établissent à $2,54 / \mathrm{m}^{2}$ en ce qui concerne $P$. perniciosus et $0,41 / \mathrm{m}^{2}$ en ce qui concerne $P$. mascittii. Ces chiffres sont intéressants à comparer avec ceux que fournissent Rioux et coll. C'est ainsi qu'en 1965, sur $235 \mathrm{~m}^{2}$ de piégeage, une densité de deux $P$. perniciosus par mètre carré a été établie, c'est-à-dire un chiffre très semblable. Par contre, les résultats obtenus par Croset en Tunisie, dans une zone écologiquement très différente, sont beaucoup plus élevés $\left(192 / \mathrm{m}^{2}\right.$ à l'étage de l'oléo-lentisque, $148 / \mathrm{m}^{2}$ à l'étage du chêne vert). En ce qui concerne $P$. mascittii, la densité est même plus forte en Touraine, puisque, de 1961 à 1966, seuls 27 exemplaires ont été capturés dans le Midi. Il apparaît donc que la très importante différence de densité globale en Phlébotomes entre le Midi et la Touraine tient uniquement à l'absence de l'espèce $P$. ariasi dans cette dernière région, $P$. perniciosus et $P$. mascittii se retrouvant en quantités équivalentes dans les deux piégeages.

L'examen du sex-ratio vient confirmer la similitude des résultats en ce domaine : Rioux et coll., en 1965, obtiennent $97 \%$ de $\sigma^{*}$ en ce qui concerne P. perniciosus, tandis que notre prospection en recueille $89 \%$. Il faut voir là une distorsion due à la technique de piégeage, et variable selon les espèces, puisque, en ce qui concerne P. mascitti, ce sont les $q$ qui dominent, avec $66 \%$.

Tout en nécessitant beaucoup de prudence, à cause du faible nombre des captures, ces résultats globaux permettent donc d'intéressantes comparaisons. Cependant, compte tenu de l'hétérogénéité des zones de piégeages sur le plan biogéographique, ils doivent être détaillés.

Sur 177 stations, 56 seulement ont révélé la présence de Phlébotomes. Bien entendu, ce résultat ne signifie pas que les autres n'abritaient pas ces Insectes, car les conditions de piégeage sont essentiellement différentes d'une station à l'autre, dépendant beaucoup de la qualité des biotopes piégeables. Il s'agit là d'un des défauts inhérents à la technique utilisée. En particulier, la présence de barbacanes est extrêmement favorable à la capture des Phlébotomes. Le tableau $I$ montre de manière significative la différence de rendement entre les piégeages effectués dans les barbacanes et les autres piégeages.

43 stations ont révélé la présence de $P$. perniciosus et 25 celle de $P$. mascittii. 12 stations hébergeaient l'une et l'autre espèce. Si l'on se rapporte aux chiffres globaux de capture, on arrive à une moyenne de 7,5 $\mathrm{P}$. perniciosus et de $2 \mathrm{P}$. mascittii par station positive. Ce chiffre illustre déjà l'extrême dilution de la population de 
TABleat: I. - Résultats comparatifs des piégages dans les barbacanes et dans les autres biotopes.

$$
\text { Biotopes }
$$

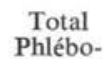

Phlébotomes

Tous biotopes confondus

Barbacanes, toutes zones

Hors barbacanes, toutes zones

Vallée du Loir, tous piégeages

Vallée du Loir, barbacanes

Vallée du Loir, hors barbacanes

$\begin{array}{rcr}378 & 128 & 2,95 \\ 369 & 78,40 & 4,71 \\ 9 & 49,60 & 0,18 \\ 130 & 13,84 & 9,50 \\ 127 & 6,84 & 18,57 \\ 3 & 7 & 0,43\end{array}$

$P$. mascittii dans la région. Il doit pourtant être corrigé en ce qui concerne $P$. perniciosus: comme l'indique le tableau II, certaines stations se sont avérées riches en $P$. perniciosus, avec les conséquences épidémiologiques que cela peut impliquer, alors que rien de semblable n'apparaît pour $P$. mascittii.

Malgré le caractère très artificiel de cette présentation et dans un but de commodité, les stations ont été classées par départements :

\section{INDRE-ET-LOIRE :}

Ballan :

$$
\begin{aligned}
& P \text {. perniciosus .... } 1 \sigma^{\star} \\
& \text { P. mascittii ............ } 2
\end{aligned}
$$

Beauchêne-la-Rudanaie :

$$
\text { P. mascittii } \ldots \ldots \ldots \ldots \ldots .1 q
$$

Benais :

$$
\text { P. perniciosus } \ldots \ldots \ldots \ldots \ldots 1 q
$$

Chinon :

$$
\text { P. perniciosus ... } 1 \sigma^{\star}
$$

Cinq-Mars

$$
\text { P. perniciosus ... } 7 \sigma^{7}
$$

Coulaines :

$$
\begin{aligned}
& \text { P. perniciosus ... } 3 \sigma^{*} \\
& \text { P. mascittii .............. } 1 \text { 우 }
\end{aligned}
$$

Courcelles-de-Touraine :

$$
\text { P. perniciosus } \ldots .44 \sigma^{*} \ldots 1 \%
$$

Fondettes :

$$
\text { P. perniciosus ... } 1 \sigma^{*}
$$

Huismes :

$$
\text { P. perniciosus ... } 48
$$

Ingrandes-de-Touraine :

$$
P \text {. perniciosus ... } 3 \delta^{*} \ldots .1 \text { q }
$$

Isle-Bouchard :

$$
P \text {. perniciosus ... } 2 \delta
$$

Joué-les-Tours :

Langeais :

$$
\begin{aligned}
& P \text {. perniciosus .... } 2 \text { के }
\end{aligned}
$$

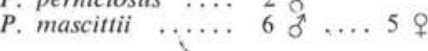

$$
\text { P. perniciosus } \ldots .66 \%
$$

Luynes :

$$
\text { P. perniciosus ... } 1 \sigma^{*}
$$

Marcilly :

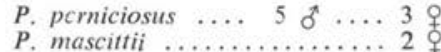

Montbazon :

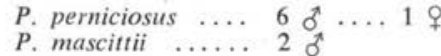

Montgauger :

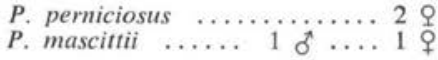

Neuil :

$$
\text { P. perniciosus } \ldots .1 \%
$$

Reugny :

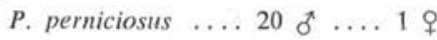

Rillé :

$$
\text { P. perniciosus .... } 4 \sigma^{\pi}
$$

Rivière :

$$
\text { P. perniciosus }
$$

Rouziers-en-Touraine :

P. mascittii 
Saint-Cyr-les-Tours :

$$
\text { P. perniciosus .... } 2 \delta^{\circ} \ldots 2 q
$$

Saint-Epain :

$$
\text { P. perniciosus ... } 1 \sigma^{*}
$$

Saint-Etienne-de-Chigny :

$P$. perniciosus

Sainte-Maure :

$$
\text { P. perniciosus .... } 3 \sigma^{*}
$$

Saint-Patrice :

$$
\text { P. perniciosus ... } 12 \sigma^{\circ}
$$

\section{LOIR-ET-CHER :}

Chalay :

$$
\text { P. mascittii } \ldots \ldots \ldots \ldots \ldots 1 q
$$

Couture-sur-Loir :

$$
\text { P. perniciosus .... } 1 \sigma
$$

La Chapelle-Enchérie :

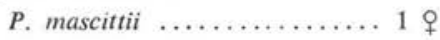

Les Roches:

$$
\begin{aligned}
& P \text {. perniciosus } \ldots . \quad 5 \sigma^{2} \ldots 1 \text { 우 } \\
& \text { P. mascittii ..... } 2 \text { के ... } 4 \text { क }
\end{aligned}
$$

Montoire :

$$
\begin{aligned}
& \text { P. perniciosus ... } 23 \delta^{\circ} \ldots .3 \text { 우 } \\
& \text { P. mascittii ............ } 1 \text { \% }
\end{aligned}
$$

\section{MAINE-ET-LoIRe :}

Baugé :

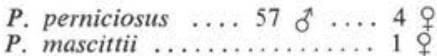

Beaufort-en-Vallée :

$$
P \text {. perniciosus ... } 1 \frac{1}{\sigma}
$$

Blou :

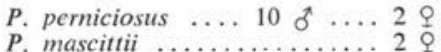

Breil :

$$
\text { P. mascittii ..... } 1 \delta
$$

\section{SARTHE :}

Beaumont-Pied-de-Bœuf :

$$
\text { P. mascittii } \ldots \ldots \ldots \ldots \ldots 1 q
$$

Château-du-Loir :

P. perniciosus
Saint-Symphorien-les-Ponceaux :

$$
P \text {. perniciosus ... } 1 \delta
$$

Semblançay :

$$
\text { P. perniciosus ... } 1 \sigma^{*}
$$$$
\text { P. mascittii .............. } 2 \text { ? }
$$

Sonzay :

$$
\text { P. mascittii } \ldots \ldots \ldots \ldots \ldots \ldots 1 \text { \% }
$$

Tours :

$$
\text { P. perniciosus .... } 1 \delta
$$

Ussé :

$$
\text { P. mascittii ..... } 3 \sigma^{7} \ldots 2 \text { \% }
$$

Périgny :

$$
\text { P. mascittii }
$$

Saint-Cyr-du-Gault :

$$
\text { P. mascittii }
$$

Saint-Quentin-les-Troo :

$$
\text { P. perniciosus } \ldots .1 \sigma^{\star} \ldots 1 \text { \& }
$$

Selommes :

$$
\text { P. mascittii ..... } 2 \sigma^{\circ}
$$

Sougé :

$$
\begin{array}{lllllll}
\text { P. perniciosus } & \ldots & 3 & 0 & \ldots & 2 & 9 \\
P . & \text { mascittii } & \ldots & \ldots & \ldots & \ldots & 1
\end{array}
$$

Villier-sur-Loir :

P. mascittii

Cheviré-le-Rouge :

$$
P \text {. perniciosus .... } 2 \text { के }
$$

Jarzé :

$$
\text { P. perniciosus ... } 11 \sigma^{\wedge} \ldots 1 \text { \% }
$$

Montigné-les-Rairies

$$
P \text {. perniciosus .... } 2 \sigma^{\circ}
$$

Saint-Georges-du-Bois :

$$
P \text {. perniciosus .... } 4 \text { के }
$$

Vernantes :

$$
\text { P. perniciosus .... } 2 \sigma^{\gamma} \ldots 1 \text { 우 }
$$

Jupille :

$$
\text { P. mascittii } \ldots \ldots \ldots \ldots \ldots \ldots 1 \text { 우 }
$$

Luché-Pringé :

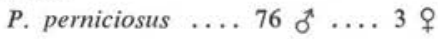




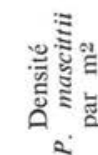

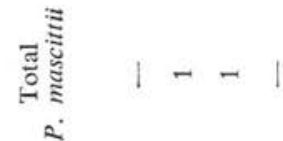

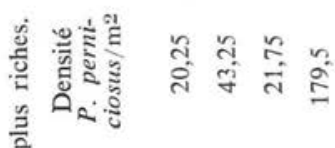

s

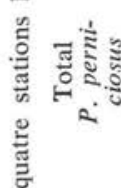

y

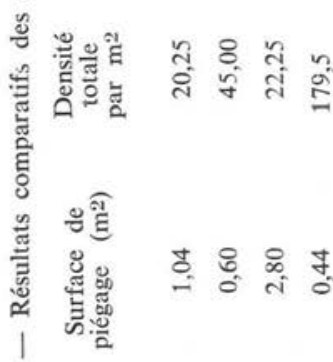

घ

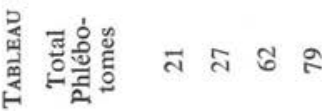

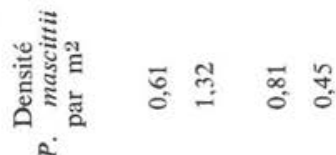

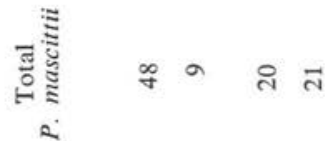

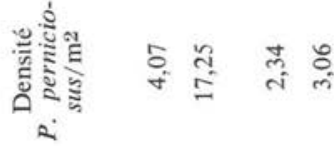

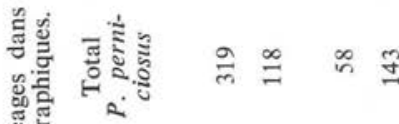

80

a

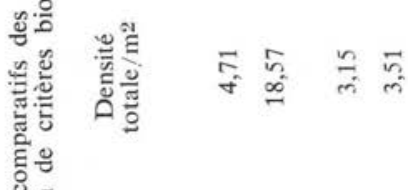

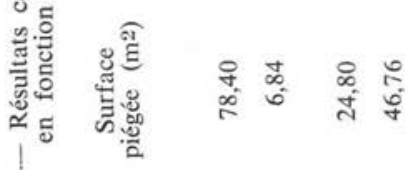

$\dot{\Xi}$

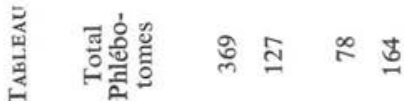

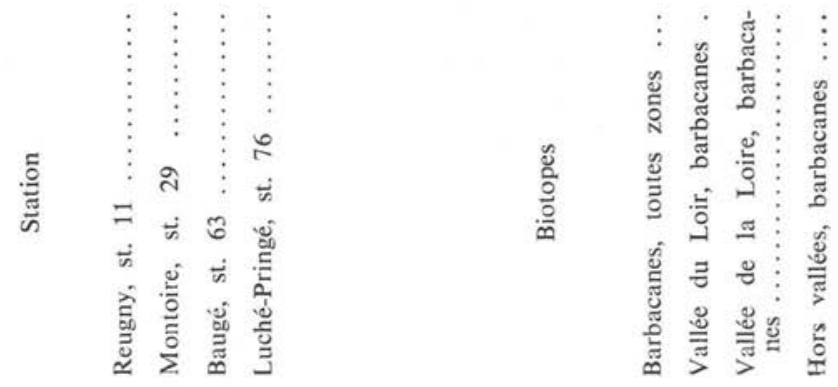


L'examen de la carte de répartition des stations positives (fig. 1) montre que leur disposition n'est pas quelconque : malgré un piégeage débordant largement l'aire de répartition du chêne pubescent en peuplements denses, la presque totalité des stations positives en $P$. perniciosus est située à l'intérieur de cette zone.

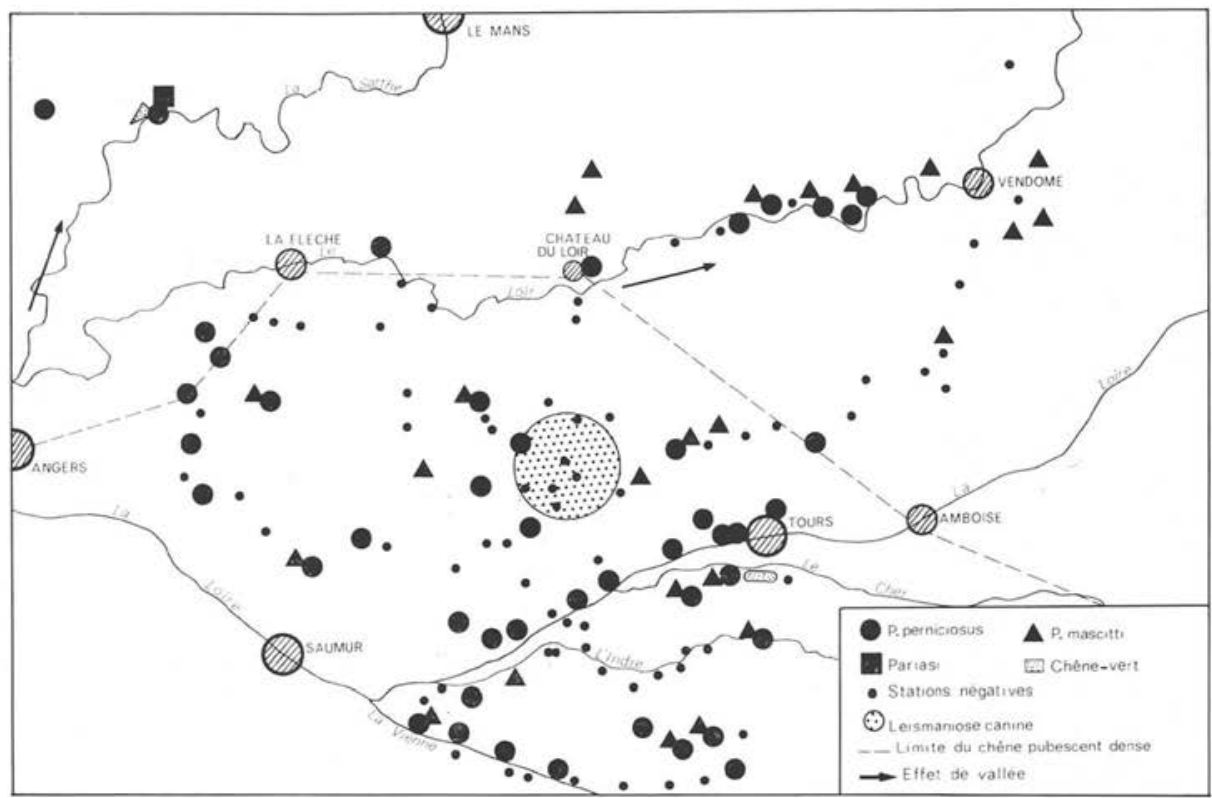

FIG. 1. - Répartition des piégeages et des captures en Touraine (juillet-août 1974).

Il existe toutefois deux exceptions à cette règle, constituées par les vallées de la Sarthe et du Loir. Nous ne reprendrons pas ici le problème de la vallée de la Sarthe, qui n'a pas été échantillonnée au cours de cette prospection, et devra faire l'objet d'une nouvelle étude. Par contre, la vallée du Loir s'est révélée très riche en Phlébotomes, ainsi que l'objectivent aussi bien le nombre des stations positives (fig. 1) que le tableau III. La densité par mètre carré de piège y est quatre fois plus importante que dans la moyenne des stations, et cette différence porte essentiellement sur $P$. perniciosus. Il faut voir là l'effet de conditions particulières, l'existence d'une falaise orientée vers le sud créant un microclimat nettement plus favorable que sur le plateau environnant. La vallée de la Loire, bien que située dans la zone du chêne pubescent et bien que présentant aussi un coteau exposé au sud, ne manifeste pas cette augmentation de densité, ainsi que le prouvent les chiffres du tableau III (1).

(1) Le faible nombre des captures ne permet malheureusement pas une étude statistique rigoureuse; néanmoins, la comparaison des variances concernant les piégages dans les barbacanes est évocatrice : 18,5 dans la vallée du Loir; 1 dans la vallée de la Loire ; 0,85 en dehors des vallées. 
La répartition de $P$. mascittii, par contre, n'est en rien tributaire des facteurs écologiques dont témoigne la répartition du chêne pubescent. Dix stations en effet sont extérieures à l'aire de peuplement dense et, parmi elles, six ne sont pas situées dans les vallées. Ceci confirme bien ce que l'on sait des exigences écologiques de l'espèce et de son aptitude à coloniser, en faible abondance, des régions beaucoup plus septentrionales que celles auxquelles se limitent les autres espèces.

Malgré tous les efforts déployés, les piégeages concernant plus particulièrement la zone où a été dépistée la leishmaniose n'ont livré que peu de Phlébotomes. Il faut voir là un défaut de la méthode de piégeage, qui n'est efficace que dans les gîtes particuliers que sont les barbacanes (tableau I). Or cette zone, sans relief, est très pauvre en constructions pourvues de ce type d'anfractuosités. D'autres piégeages, reposant sur d'autres techniques, devraient permettre d'obvier à cet inconvénient. Il sera par contre intéressant d'orienter les études à venir sur la leishmaniose canine vers les zones trouvées riches en Phlébotomes, et tout particulièrement vers la vallée du Loir, pour voir si des différences se révèlent dans l'intensité de l'infestation.

\section{Conclusion}

Dans une zone connue comme abritant un foyer de leishmaniose canine, mais très septentrionale, cette prospection systématique a permis de mettre en évidence la présence d'un vecteur de la maladie, en quantité non négligeable. Phlebotomus perniciosus se présente ainsi comme le responsable probable de la persistance de cette enzootie. Anthropophile, il est parfaitement capable de transmettre l'affection à l'homme, et des cas sporadiques de leishmaniose humaine sont à redouter.

Dans la mesure où le nombre des piégeages permet de le dire, il semble que la répartition de l'espèce obéisse aux mêmes critères en Touraine et dans le Midi, $P$. perniciosus disparaissant dans les stations situées en dehors de l'aire de répartition du chêne pubescent en peuplements denses. Seul persiste alors $P$. mascittii, certes capable de transmettre la leishmaniose, mais toujours très peu abondant. Cette situation est ceile du plateau beauceron, tel qu'il a été échantillonné dans la zone nord-est de la prospection. C'est aussi celle qui avait été trouvée, en 1971, lors d'une étude effectuée plus au nord, dans la vallée de l'Eure, à propos de cas sporadiques trouvés dans un chenil.

Il apparaît donc que le foyer étudié se situe probablement à l'extrême limite septentrionale de la zone dans laquelle un vecteur potentiel de la leishmaniose peut vivre en abondance suffisante pour entretenir une enzootie. Ainsi se trouvent une fois encore confirmé le rôle d' "élément focalisateur " du vecteur dans le cas des leishmanioses, et justifiées les études détaillées nécessaires à sa meilleure connaissance. 


\section{Bibliographie}

1. Castillon (M.), 1937. - Le Kala-azar en France. Rev. Serv. Santé mil., 106, 701-750.

2. Clatsse (R.), Di Matteo (J.), Deuil (R.) et De Traverse (P. M.), 1950. - Diabète aigu après Kala-azar traité par la Diamidine. Bull. Mem. Soc. med. Hôp. Paris, 153-57.

3. Croset (H.), 1969. - Ecologie et systématique des Phlebotomini (Diptera, Psychodidae) dans deux foyers, français et tunisien, de leishmaniose viscérale. Essai d'interprétation écologique. Thèse doct. ès sciences, Montpellier, $646 \mathrm{p}$.

4. Domergue (G.), 1950. - De la leishmaniose viscérale rhodanienne (à propos d'un cas observé à Grenoble). Thèse doct. méd., Lyon.

5. Guilhon (J.), 1950. - Répartition géographique de la leishmaniose canine en France. Bull. Acad. vét., 24, 69-74.

6. Guilhon (J.), 1950. - Un nouveau cas de leishmaniose canine autochtone dans la région parisienne. Bull. Acad. vét., 23, 361-62.

7. Guilhon (J.), 1951. - Leishmaniose canine et antigène tuberculeux. Bull. Acad. vét., 24, 523-24.

8. Guil.hon (J.) et Loge (G.), 1950, -- Leishmaniose canine autochtone en Bretagne. C.R. Acad. Sci., 230, 274-78.

9. Hany (Y.), Allanic (H.) et Vivien (P.), 1974. - Le Kala-azar de l'adulte dans l'ouest de la France au cours des 15 dernières années. Bull. Soc. Path. exot., 67, 503-12.

10. Henry (A.) et Bory (L.), 1935. - Un cas de leishmaniose canine dans la région parisienne. Question prophylactique. Bull. Soc. Dermato-Syph., 42, 928-30.

11. Houin (R.), 1962. - Contribution à l'étude des leishmanioses autochtones en France métropolitaine. Thèse doct. méd., $116 \mathrm{p}$.

12. HouIN (R.), 1963 - Données épidémiologiques et déductions prophylactiques sur les leishmanioses autochtones en France. Ann. Paras. hum. comp., 38, 379-438.

13. Houin (R.) et Beaucournu (J.-C.), 1966. -- Nouvelles stations françaises de Phlébotomes. Ann. Paras. hum comp., 41, 387-90.

14. Houin (R.), Jolivet (G.), Combescot (C.), Deniau (M.), Puel (F.), Barbier (D.), Romano (P.) et Kerbeuf (D.), 1974. - Etude préliminaire d'un foyer de leishmaniose canine dans la région de Tours. C.R. Colloque international sur l'écologie des leishmanioses, Montpellier (à paraître).

15. Kerbeuf (D.), 1973. - Diagnostic immunologique de la leishmaniose canine. Etude de la méthode d'immunofluorescence et application à une enquête épidémiologique dans la région de l'ouest. Thèse méd.-vét. Alfort, 161 p., 8 fig.

16. Langeron (M.) et Nitzulescu (V.), 1932, - Révision des Phlébotomes de France. Ann. Paras. hum. comp., 10 (3), 286-94.

17. Lavier (G.), 1937. - Localité française nouvelle pour Phlebotomus perniciosus. Ann. Paras. hum. comp., 15, 91.

18. Nitzulescu (V.) et Dollfus (R.), 1934. - Présence à Richelieu (Indre-et-Loire) de Phlebotomus perniciosus Newstead. Ann. Paras. hum. comp., 12, 69.

19. Parrot (L.), 1936. - Présence de Phlebotomus perniciosus dans le département de l'Indre. Arch. Inst. Pasteur Algérie, 14, 427. 
20. Pehu (M.) et Bertoye (P.), 1931. - Sur un cas de Kala-azar survenu chez un enfant de cinq ans n'ayant jamais quitté la région des Vosges. Lyon méd., 167, 731-34.

21. Pehu (M.) et Bertoyf (P.), 1937. - Trois cas non-méditerranéens de Kala-azar infantile autochtone: Vosges, Ardèche, Isère. Bull. Acad. méd., 118, 342-44.

22. Prat (J.), 1944. - Sur un cas autochtone de leishmaniose canine observé dans le département de l'Allier. Rev. Méd. vét., 95, 16-25.

23. Rioux (J. A.) et coll., 1969. - Epidémiologie des leishmanioses dans le sud de la France. Monographie de l'INSERM, $\mathrm{n}^{\circ} 37,223 \mathrm{p}$.

24. Rageau (J.), 1948. - Observations sur les Phlébotomes de la région de Poitiers. Bull. Soc. Path. exot., 41 (3-4), 217-22. 